\title{
ROS Generated by Upconversion Nanoparticle-Mediated Photodynamic Therapy Induces Autophagy via PI3K/AKT/ mTOR Signaling Pathway in M1 Peritoneal Macrophage
}

\author{
Xiaobo Han ${ }^{\mathrm{a}}$ Jiayuan Kou ${ }^{\mathrm{b}} \quad$ Yinghong Zheng $^{\mathrm{a}} \quad$ Zhongni Liu $^{\mathrm{a}} \quad$ Yueqing Jiang $^{\mathrm{a}}$ \\ Ziyu Gao ${ }^{a}$ Lin Cong ${ }^{a}$ Liming Yang ${ }^{a}$ \\ aDepartment of Pathophysiology, Key Laboratory of Cardiovascular Pathophysiology, Harbin Medical \\ University, Harbin, China, b Department of Biochemistry and Molecular Biology, Harbin Medical \\ University, Harbin, China
}

\section{Key Words}

Reactive oxygen species $•$ Autophagy $\cdot M 1$ Peritoneal macrophage $\cdot$ Upconversion nanoparticle - Photodynamic therapy

\begin{abstract}
Background/Aims: Atherosclerosis is a chronic inflammatory cardiovascular disease. Macrophages are major components of atherosclerotic plaques and play a key role in the development of atherosclerosis by secreting a variety of pro-inflammatory factors. Our previous studies have confirmed that upconversion nanoparticles encapsulating chlorin e6 (UCNPs-Ce6) mediated photodynamic therapy (PDT) can promote cholesterol efflux and induce apoptosis in THP-1 macrophages. In this study, we investigated whether reactive oxygen species (ROS) generated by UCNPs-Ce6-mediated PDT can induce autophagy to inhibit the expression of pro-inflammatory factor in M1 peritoneal macrophages. Methods: Peritoneal macrophages were collected from C57/BL6 mice injected with 3\% thioglycollate broth medium and induced by lipopolysaccharides and interferon- $\gamma$. Intracellular ROS production was assessed by 2'-7'-dichloroflorescein diacetate and flow cytometry. Autophagy was assayed by western blot, transmission electron microscopy and immunofluorescence. Pro-inflammatory cytokines were detected by enzyme-linked immunosorbent assay and western blot. Results: Model M1 peritoneal macrophages were established after $24 \mathrm{~h}$ induction. Protein expression levels of LC3 II and Beclin1, and degradation of p62 increased and peaked at $2 \mathrm{~h}$ in the PDT group. Meanwhile, levels of inflammatory cytokines iNOS, IL-12, and TNF- $\alpha$ markedly decreased after PDT. The increase in autophagy levels and decrease in pro-inflammatory cytokines were significantly inhibited by 3-methyladenine. Furthermore, ROS generated by UCNPs-

X. Han, J. Kou and Y. Zheng contributed equally to this work.

$\begin{array}{ll}\text { Liming Yang } & \text { Department of Pathophysiology, Harbin Medical University } \\ & \text { Harbin (China) } \\ & \text { Tel. }+8645186674548 \text {, Fax }+8645186674548 \text {, E-Mail limingyang@ems.hrbmu.edu.cn }\end{array}$
\end{abstract}




\section{Cellular Physiology Cell Physiol Biochem 2019;52:1325-1338

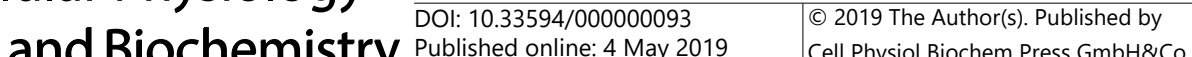 \\ \begin{tabular}{l|l} 
Published online: 4 May 2019 & Cell Physiol Biochem Press GmbH\&Co. KG
\end{tabular} \\ Han et al.: Upconversion Nanoparticle-Mediated Photodynamic Therapy Induces \\ Autophagy in M1 Peritoneal Macrophage}

Ce6 mediated PDT activated autophagy. The expression of autophagy related-protein and inflammatory cytokines iNOS, IL-12, and TNF- $\alpha$ were inhibited by the ROS inhibitor N-acetyl cysteine. Conclusion: ROS generated by UCNPs-Ce6-mediated PDT activated autophagy and inhibited the expression of pro-inflammatory factors of $\mathrm{M} 1$ peritoneal macrophage via the $\mathrm{PI} 3 \mathrm{~K} / \mathrm{AKT} / \mathrm{mTOR}$ signaling pathway.

(c) 2019 The Author(s). Published by Cell Physiol Biochem Press GmbH\&Co. KG

\section{Introduction}

Atherosclerosis is a common cardiovascular disease, and a serious threat to health, and it is generally believed that inflammation is the main cause. Macrophages are involved in the progression of the atherosclerosis [1-3]. Macrophages are mainly divided into pro-inflammatory M1 macrophages and anti-inflammatory M2 macrophages, and their proportion in atherosclerosis plaque changes dynamically with the development of the disease. M1 macrophages secrete pro-inflammatory factors causing unstable plaques, which eventually rupture and cause clinical symptoms $[4,5]$. Therefore, by inhibiting the expression of pro-inflammatory factors secreted by M1 macrophages, plaque inflammation can be reduced and plaque stability increased $[5,6]$.

Autophagy is involved in the metabolism of proteins, glucose, and lipids, and is central to intracellular homeostasis and development $[7,8]$. Furthermore, autophagy dysfunction is closely associated with cardiovascular disease $[9,10]$. Numerous studies have demonstrated that autophagy plays an important anti-inflammatory role in atherosclerosis [11-13]. Deficient macrophage autophagy increases plaque instability by activating inflammation, which suggests that intact autophagy processes are essential for reducing the occurrence of adverse vascular events through the suppression of inflammation $[14,15]$.

Photodynamic therapy (PDT) is a new therapeutic strategy for cardiovascular disease and is non-invasive, accurate, and has few side effects. PDT mainly involves three important components: the photosensitizer, light and oxygen molecules [16-19]. The photosensitizer is a vital component of PDT. Accordingly, we chose a composite photosensitizer UCNPs-Ce6 as a medium, which can be used with reasonable efficiency together with a $980 \mathrm{~nm}$ laser $[20,21]$. In contrast to Photofrin, 5-aminolevulinic acid, and even third-generation photosensitizers, which are activated by visible light $(660 \mathrm{~nm})$, the UCNP-Ce6 photosensitizer is activated by near-infrared (NIR) light $(980 \mathrm{~nm})$. The penetration depth of NIR light $(980 \mathrm{~nm})$ is deeper than that of the visible light $(660 \mathrm{~nm})$. UCNPs-Ce6 increases the penetration depth of laser during PDT $[22,23]$. Our prior studies have confirmed that PDT can promote cholesterol efflux by inducing autophagy and induce THP-1 macrophage apoptosis to attenuate the progression of atherosclerosis [24-26]. This provides an important basis for UCNPs-Ce6 mediated PDT to inhibit the expression of inflammatory factor in this study.

Here, we investigated whether UCNPs-Ce6-PDT could induce autophagy to inhibit inflammation by the generation of reactive oxygen species (ROS) via the PI3K/AKT/mTOR signaling pathway in M1 peritoneal macrophages.

\section{Materials and Methods}

980-nm PDT device

The 980-nm PDT device was developed by the Harbin Institute of Technology (Harbin, China). The laser generator emits single 980-nm wavelength irradiation. For PDT treatment, the distance from the cell to the laser generator is $2 \mathrm{~cm}$ with a laser irradiation density of $1.5 \mathrm{~W} / \mathrm{cm}^{2}[27,28]$. 


\section{Cellular Physiology Cell Physiol Biochem 2019;52:1325-1338 \begin{tabular}{l|l} 
and Biol: 10.33594/000000093 & 2019 The Author(s). Published by
\end{tabular} and BIOChemistry Published online: 4 May $2019 \quad$ Cell Physiol Biochem Press GmbH\&Co. KG \\ Autophagy in M1 Peritoneal Macrophage}

\section{PDT treatment protocols}

UCNPs-Ce6 was designed by the Harbin Institute of Technology. Approximately $1 \mathrm{mg} / \mathrm{mL}$ of stock solution in dimethyl sulfoxide was stored at $4{ }^{\circ} \mathrm{C}$ in the dark.

For the inhibition studies, $1 \mathrm{mM}$ of the ROS inhibitor N-acetyl cysteine (NAC; Sigma-Aldrich, St. Louis, MO, USA), $10 \mathrm{mM}$ of the autophagy inhibitor 3-methyladenine (3-MA; Sigma-Aldrich), $10 \mu \mathrm{M}$ of the PI3K inhibitor LY294002 (Sigma-Aldrich) and $20 \mathrm{mM}$ of the AKT inhibitor triciribine (Sigma-Aldrich) were incubated together with UCNPs-Ce6 60 min before UCNPs-Ce6-mediated PDT.

\section{Cell culture}

Peritoneal macrophages were collected from C57/ BL 6 mice injected with $2 \mathrm{~mL}$ of $3 \%$ thioglycollate broth medium for $72 \mathrm{~h}$. The cells were cultured in RPMI 1640 medium (HyClone, Laboratories Inc., South Logan, UT, USA) containing 10\% fetal bovine serum (FBS; HyClone, Laboratories Inc), and $20 \mu \mathrm{g} / \mathrm{mL}$ of penicillin and streptomycin (Sigma-Aldrich, USA) for $24 \mathrm{~h}$. Cells were maintained at $37^{\circ} \mathrm{C}$ in a humidified incubator with $5 \% \mathrm{CO}_{2}$. For the experiments, the cells were seeded in 35 -mm Petri dishes or 96 -well plates at a density of $1.0 \times 10^{5}$ cells $/ \mathrm{mL}$. For M1 induction, $100 \mathrm{ng} / \mathrm{mL}$ of lipopolysaccharide (LPS; Sigma-Aldrich) and $20 \mathrm{ng} / \mathrm{mL}$ of interferon- $\gamma$ (IFN- $\gamma$; PeproTech, Rocky Hill, NJ, USA) were added into the culture medium for $24 \mathrm{~h}$. Cells were then collected, differentiated and randomly divided into four groups: (1) control, (2) laser alone, (3) UCNPs-Ce6 alone, (4) PDT.

\section{Cell viability assay}

The cell survival rate was detected by the Cell Counting Kit 8 (CCK-8) assay (Beyotime Biotechnology, Jiangsu, China). M1 peritoneal macrophages were seeded in 96-well culture plates. The plate was carefully washed with phosphate-buffered saline (PBS) and $100 \mu \mathrm{L}$ medium containing CCK-8 (RPMI 1640 medium and CCK-8 volume ratio of 9:1) was added into the wells after the different treatments. After incubation for $30 \mathrm{~min}$ at $37^{\circ} \mathrm{C}$ in the dark, the absorption of each well was measured by a microplate reader (Varian Australia Pty Ltd, Melbourne, Australia) at $450 \mathrm{~nm}$. All experiments were repeated three times.

\section{Detection of intracellular ROS}

Intracellular ROS were analyzed by $2^{\prime}$ - $7^{\prime}$-dichloroflorescein diacetate (DCFH-DA; Beyotime Biotechnology). After PDT, the cells were washed with PBS and incubated with $20 \mu \mathrm{M}$ DCFH-DA diluted in RPMI 1640 medium for $30 \mathrm{~min}$ at $37^{\circ} \mathrm{C}$ in the dark. Cells were then washed with PBS, and then measured under a fluorescence microscope (Olympus IX81, Olympus, Tokyo, Japan) at $488 \mathrm{~nm}$ excitation and $525 \mathrm{~nm}$ emission wavelengths. For the flow cytometry assay, cells were analyzed using a flow cytometer (BectonDickinson, Franklin Lakes, NJ, USA). All experiments were repeated three times.

\section{Transmission electron microscopy}

Following different treatments, the cells were harvested by centrifugation and fixed with $2.5 \%$ glutaraldehyde at $4{ }^{\circ} \mathrm{C}$ overnight. Cells were then measured by transmission electron microscopy (JEM1220, JEOL, Tokyo, Japan). All experiments were repeated three times.

\section{Fluorescence staining}

After the treatments, cells were washed with PBS, and incubated with $500 \mu \mathrm{g} / \mathrm{mL}$ CD16/32 and tumor necrosis factor (TNF- $\alpha$; Affymetrix eBioscience, USA) for $30 \mathrm{~min}$ at $37^{\circ} \mathrm{C}$ in the dark. After washing with PBS, the cells were observed under a fluorescence microscope (Olympus IX81). All experiments were repeated three times.

\section{Immunofluorescence staining}

At the indicated times after PDT, cells were washed with PBS, and fixed with $4 \%$ paraformaldehyde for $30 \mathrm{~min}$ and permeabilized with $1 \%$ Triton X-100 for $20 \mathrm{~min}$ at room temperature. Cells were washed with PBS, blocked with 3\% bovine serum albumin, and incubated with Lamp2 and LC3 antibodies (1:200) overnight at $4{ }^{\circ} \mathrm{C}$. Cells were again washed with PBS and incubated with the corresponding secondary antibody at $37^{\circ} \mathrm{C}$ temperature for $1 \mathrm{~h}$ in the dark. After washing with PBS, the cells were observed by laser scanning confocal microscope (LSCM; Meta, Germany). All experiments were repeated three times. 


\section{Cellular Physiology Cell Physiol Biochem 2019;52:1325-1338 \begin{tabular}{l|l} 
and Bincl $10.33594 / 000000093$ & 2019 The Author(s). Published by
\end{tabular} and Biochemistry \\ Han et al.: Upconversion Nanoparticle-Mediated Photodynamic Therapy Induces \\ Autophagy in M1 Peritoneal Macrophage}

\section{Monodansylcadaverine staining}

Monodansylcadaverine (MDC; Sigma-Aldrich) was used to observe the autophagic vacuole. After the different treatments, cells were incubated with $50 \mu \mathrm{mol} / \mathrm{mL} \mathrm{MDC}$ for $30 \mathrm{~min}$ at $37{ }^{\circ} \mathrm{C}$ in the dark. After washing with PBS, the cells were observed under a fluorescence microscope (Olympus IX81). All experiments were repeated three times.

Acridine orange staining

Acridine orange staining (Sigma-Aldrich) was used to observe acidic vesicle organelles. At $2 \mathrm{~h}$ after the different treatments, the cells were incubated with $0.01 \%$ acridine orange for $30 \mathrm{~min}$ at $37{ }^{\circ} \mathrm{C}$ in the dark. After washing twice with PBS, the cells were observed under a fluorescence microscope (Olympus IX81). All experiments were repeated three times.

\section{Western blot analysis}

After the treatments, RIPA lysis buffer was used to extract the total protein. Protein concentrations were assessed via a bicinchoninic acid kit (Beyotime Biotechnology). After quantification and denaturation, the protein samples were electrophoresed in sodium dodecyl sulfate-polyacrylamide gel and transferred onto a $0.45 \mu \mathrm{m}$ polyvinylidene fluoride membranes (Merck Millipore, Darmstadt, Germany). Blocking was achieved with 5\% low-fat milk diluted with Tris-buffered saline and Tween 20, and the membranes were incubated with primary antibodies at $4{ }^{\circ} \mathrm{C}$ overnight. After washing with TBST, the membranes were incubated with horseradish peroxidase (HRP) - conjugated secondary antibodies for $2 \mathrm{~h}$ at room temperature. After a further wash with TBST, the immune complexes were detected using an enhanced chemiluminescence reagent. The protein bands were quantified using Quantity One software (Bio-Rad Laboratories, Hercules, CA, USA). All experiments were repeated three times.

Antibodies against Beclin1, p62, LC3B, mTOR, p-mTOR, AKT, and p-AKT (1:1000) were purchased from Cell Signaling Technology (Boston, MA, USA). Antibodies against iNOS, IL-12 and TNF- $\alpha$ (1:1000) were purchased from Abcam (Burlingame, CA, USA). Antibodies against $\beta$-actin (1:1000) were purchased from Proteintech Group (Wuhan, China). The HRP-conjugated secondary mouse and rabbit antibodies (1:5000) were purchased from ZhongShan Company (Beijing, China).

\section{Enzyme linked immunosorbent assay}

To detect cytokines released by M1 peritoneal macrophages, cell supernatants were collected after the different treatments, and the cytokines of iNOS, IL-12 and TNF- $\alpha$ were measured by enzyme-linked immunosorbent assay (ELISA) kits (Elabscience Biotechnology Co, Ltd, Hubei, China). All experiments were repeated three times.

\section{Statistical analysis}

All experiments were repeated at least three times independently. The data were analyzed using one-way analysis of variance and the independent test. The results are presented as the mean \pm standard deviation. $P<0.05$ was considered statistically significant.

\section{Results}

\section{Establishment of M1 peritoneal macrophage model}

To establish the M1 peritoneal macrophage model, LPS and IFN- $\gamma$ were added into the culture medium with peritoneal macrophages. After induction for $24 \mathrm{~h}$, protein expression and the secretion of iNOS, IL-12, and TNF- $\alpha$ in the supernatant increased significantly (Fig. 1A, D). Subsequently, we verified the presence of specific fluorescence markers CD16/32, and TNF- $\alpha$ and pro-inflammatory factor proteins of M1 peritoneal macrophages, the expression of green fluorescence and protein level increased significantly (Fig. 1B, C). All results further confirmed the establishment of the M1 peritoneal macrophage model. 


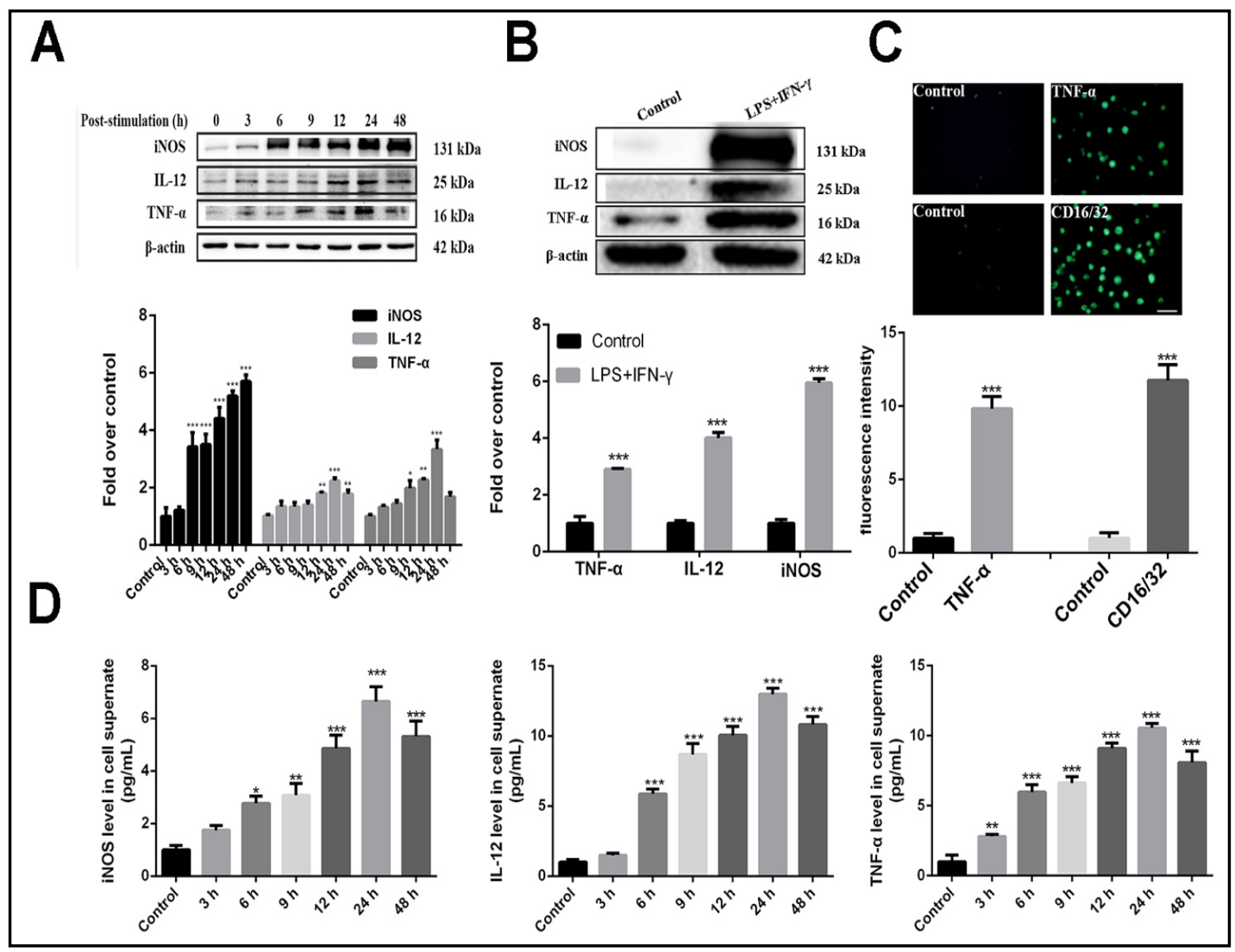

Fig. 1. Establishment of the M1 peritoneal macrophage model. (A) The expression of iNOS, IL-12 and TNF- $\alpha$ at different induction times was determined by western blotting $(n=3)$. (B) Western blot analysis of expression of iNOS, IL-12, and TNF- $\alpha$ after $24 \mathrm{~h}$ induction $(\mathrm{n}=3)$. (C) Fluorescence assay analysis of TNF- $\alpha$ and CD16/32 after induction for $24 \mathrm{~h}$ (scale bar: $50 \mu \mathrm{m})(\mathrm{n}=10)$. (D) The secretion of pro-inflammatory factors at different induction times was determined by the ELISA assay $(\mathrm{n}=10)$. ${ }^{*} \mathrm{P}<0.05$, ${ }^{* *} \mathrm{P}<0.01,{ }^{* * *}$ $\mathrm{P}<0.001$ vs control group.

Optimization of UCNPs-Ce6-mediated PDT conditions for M1 peritoneal macrophages

To choose the optimal PDT time, intensity, and photosensitizer dose, cell viability was determined with the CCK-8 assay after different treatments. By screening the different concentrations and laser intensities, a dose of $6 \mu \mathrm{g} / \mathrm{mL}$ of UCNPs-Ce 6 and a laser intensity of $1.5 \mathrm{~W} / \mathrm{cm}^{2}$ were considered safe and had no significant effect on cell viability (Fig. 2A, B). Furthermore, we tested a combination of drug concentration and time (Fig. 2C), laser intensity and time (Fig. 2D), and drug concentration and laser intensity (Fig. 2E) via CCK-8 assay. Accordingly, $6 \mu \mathrm{g} / \mathrm{mL}$ of UCNPs-Ce 6 and laser irradiation at $1.5 \mathrm{~W} / \mathrm{cm}^{2}$ of $60 \mathrm{~s}$ were selected as the optimal conditions for the M1 peritoneal macrophages (Fig. 2F).

\section{UCNPs-Ce6-mediated PDT inhibited the expression and secretion of pro-inflammatory} factors in M1 peritoneal macrophages

To investigate the effects of PDT on the pro-inflammatory factors of M1 peritoneal macrophages, the expression of pro-inflammatory factor proteins was tested at different induction times. As shown in Fig. 3A, the inflammatory factors decreased significantly at $12 \mathrm{~h}$ after PDT. Pretreatment with 3-MA increased the expression and secretion of proinflammatory factors (Fig. 3B, C). Moreover, compared with the other groups, the secretion of the pro-inflammatory factors in the supernatants of the PDT group was also significantly decreased at $12 \mathrm{~h}$ after PDT (Fig. 3D). Thus, PDT inhibited the expression of pro-inflammatory factors in M1 peritoneal macrophages. Next, we verified the specific fluorescence markers 


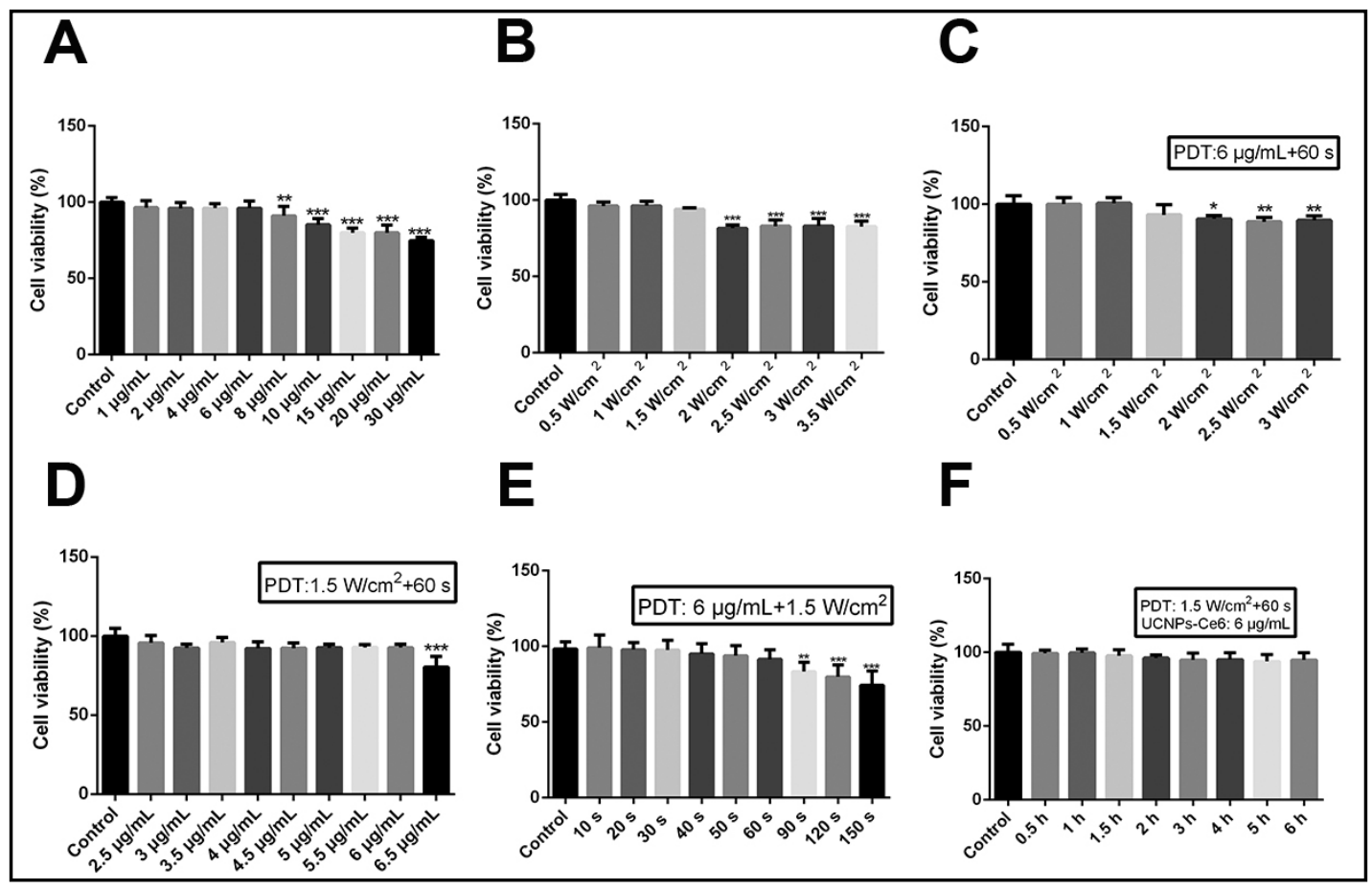

Fig. 2. Survival rates of M1 peritoneal macrophages after PDT. The survival rates of M1 peritoneal macrophages (A) following exposure to different UCNPs-Ce6 concentrations for $4 \mathrm{~h}(\mathrm{n}=10)$. (B) following exposure to different laser densities at $60 \mathrm{~s}(\mathrm{n}=10)$. (C) following exposure to different laser densities at $6 \mu \mathrm{g} / \mathrm{mL}$ UCNPs-Ce6 and $60 \mathrm{~s}(\mathrm{n}=10)$. (D) following exposure to different UCNPs-Ce6 concentrations at $1.5 \mathrm{~W} / \mathrm{cm}^{2}$ and $60 \mathrm{~s}(\mathrm{n}=10)$. (E) following exposure to different laser irradiation times at $1.5 \mathrm{~W} / \mathrm{cm}^{2}$ and 6 $\mu \mathrm{g} / \mathrm{mL}$ UCNPs-Ce6 $(\mathrm{n}=10)$. (F) following exposure to $6 \mu \mathrm{g} / \mathrm{mL}$ UCNPs-Ce6, $1.5 \mathrm{~W} / \mathrm{cm}^{2}$ and $60 \mathrm{~s}(\mathrm{n}=10) .{ }^{*}$ $\mathrm{P}<0.05,{ }^{* *} \mathrm{P}<0.01,{ }^{* * *} \mathrm{P}<0.001$ vs control group.

CD16/32 and TNF- $\alpha$ of M1 peritoneal macrophages. The intensity of green fluorescence also decreased significantly in the PDT group, and was inhibited by 3-MA (Fig. S1A, B - for all supplemental material see www.cellphysiolbiochem.com). These results suggested that PDT inhibited the expression and secretion of pro-inflammatory factors in M1 peritoneal macrophages through the activation of autophagy.

\section{UCNPs-Ce6-mediated PDT induced autophagy in M1 peritoneal macrophage}

To verify whether the UCNPs-Ce6-mediated PDT could induce autophagy in M1 peritoneal macrophages, autophagy related-proteins were examined at different time points. We found that UCNPs-Ce6-mediated PDT upregulated LC3 II and Beclin1 expression and downregulated p62 expression at $2 \mathrm{~h}$ (Fig. 4A). Subsequently, we used the autophagy broad-spectrum inhibitor 3-MA and found that LC3 II conversion, Beclin1 expression and p62 degradation were suppressed by 3-MA after PDT (Fig. 4B). In addition, autophagosome ultrastructures of M1 peritoneal macrophages were observed by transmission electron microscopy after the different treatments. Compared with the other groups, macrophages in the PDT group appeared to be typical autophagosomes with phospholipid bilayers, and this formation was inhibited by 3-MA (Fig. 4C). Moreover, the co-localization of Lamp2 and LC3 detected after PDT further confirmed autophagy (Fig. 4D). Additionally, we investigated autophagic flux after PDT with MDC and acridine orange staining. The results indicated that the green fluorescence in the PDT group was brighter and the red fluorescence was more obvious than in macrophages of the other groups (Fig. 4E, F). Therefore, UCNPs-Ce6mediated PDT induced autophagy in M1 peritoneal macrophages. 


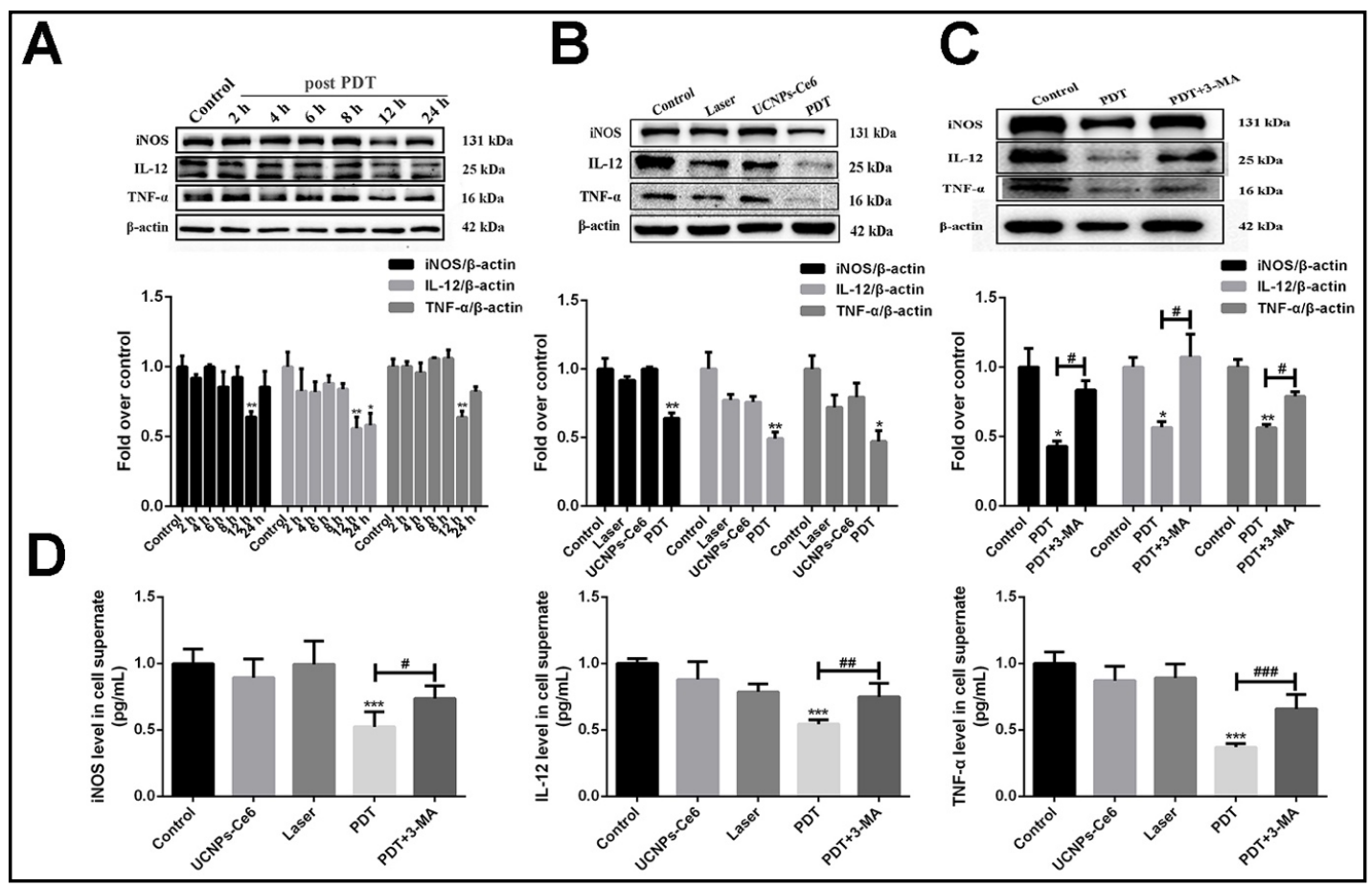

Fig. 3. PDT inhibited the expression of pro-inflammatory factors. (A) The expression of iNOS, IL-12, and TNF- $\alpha$ at different treatment times $(\mathrm{n}=3)$. (B) Western blotting analysis of iNOS, IL-12, and TNF- $\alpha$ with different treatments at $12 \mathrm{~h}(\mathrm{n}=3)$. (C) The effect of 3-MA on the expression of iNOS, IL-12, and TNF- $\alpha$ at $12 \mathrm{~h}$ after PDT $(\mathrm{n}=3)$. (D) The secretion levels of iNOS, IL-12, and TNF- $\alpha$ in cell supernatant with different treatment at $12 \mathrm{~h}(\mathrm{n}=10) .{ }^{*} \mathrm{P}<0.05,{ }^{* *} \mathrm{P}<0.01,{ }^{* * *} \mathrm{P}<0.001$ vs control group, ${ }^{\#} \mathrm{P}<0.05,{ }^{\# \#} \mathrm{P}<0.01$, ${ }^{\# \# \#} \mathrm{P}<0.001$ vs PDT group.

UCNPs-Ce6-mediated PDT induced autophagy by inhibiting the PI3K/AKT/mTOR signaling pathway in M1 peritoneal macrophages

The PI3K/AKT/mTOR signaling pathway is the classic pathway of autophagy [29]. As shown in Fig. $5 \mathrm{~A}$, the expression of p-AKT and p-mTOR had clearly decreased at $2 \mathrm{~h}$ after PDT. Additionally, changes in the expression of p-AKT and p-mTOR were not obvious, but the levels of autophagy associated protein LC3 II significantly increased after pretreatment with PI3K inhibitor LY294002 and AKT inhibitor triciribine (Fig. 5B, C). These results indicated that UCNPs-Ce6-PDT induced autophagy through the PI3K/AKT/mTOR signaling pathway.

UCNPs-Ce6-mediated PDT promoted ROS generation in M1 peritoneal macrophages and induced autophagy by suppressing the PI3K/AKT/ mTOR signaling pathway

The generation of ROS represents the foundation of PDT [30]. Compared with other groups, significant green fluorescence was observed in the PDT group with DCFH-DA staining. The increase in ROS production was inhibited by pretreatment with NAC (Fig. 6A). Furthermore, the generation of ROS was examined by flow cytometry, and ROS levels increased significantly after UCNPs-Ce6-PDT. ROS production was effectively attenuated by NAC (Fig. 6B).

Next, we investigated the effect of ROS on PDT induced autophagy. The levels of expression of autophagy-related proteins and PI3K/AKT/mTOR signaling pathway related proteins were examined by pretreatment with NAC. The results showed that the expression of Beclin1, the transformation of LC3 II and the degradation of p62 were inhibited by NAC. Simultaneously, the expression of p-mTOR and p-AKT clearly increased compared with the PDT group (Fig. 6C, D). To further validate the relationship between autophagy and ROS, acridine orange and MDC staining was used to observe the acidic vesicular organelles. 


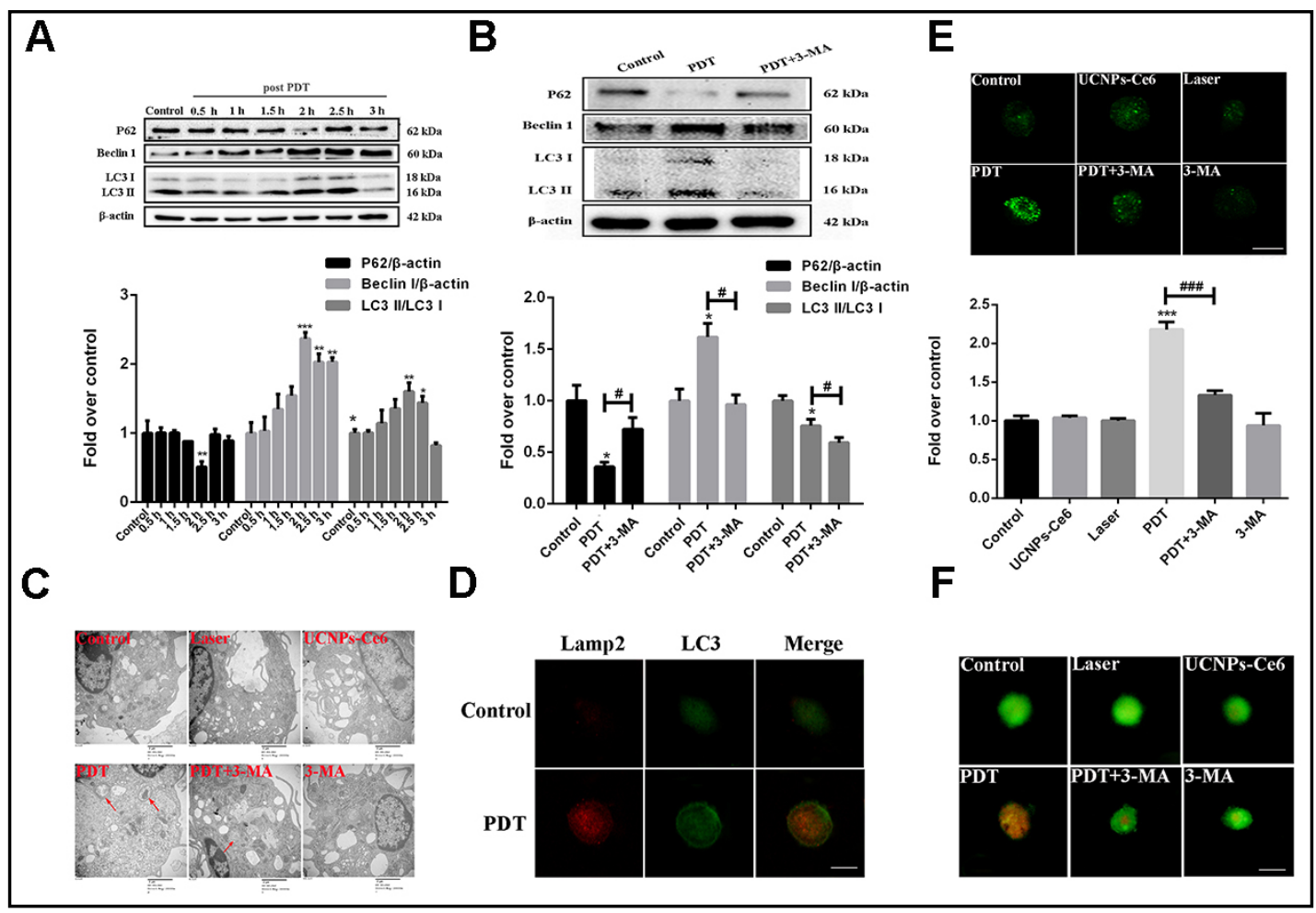

Fig. 4. PDT induced autophagy in M1 peritoneal macrophages. (A) The expression levels of the p62, Beclin1, and LC3 II/LC3 I at different treatment times after PDT $(n=3)$. (B) The effects of 3-MA on the expression of p62, Beclin1, and LC3II/LC3I ( $\mathrm{n}=3$ ). (C) Morphological changes of M1 peritoneal macrophages were observed using transmission electron microscopy. Red arrows indicate the autophagosomes (scale bar: 2 $\mu \mathrm{m})$. (D) LC3 and Lamp2 co-localization at $2 \mathrm{~h}$ after PDT using LSCM (scale bar: $5 \mu \mathrm{m}$ ). (E) Autophagic vacuoles induced by various treatments using MDC staining (scale bar: $5 \mu \mathrm{m}$ ). (F) Autophagic vacuoles induced by various treatments using acridine orange staining (scale bar: $50 \mu \mathrm{m}$ ). ${ }^{*} \mathrm{P}<0.05,{ }^{* *} \mathrm{P}<0.01,{ }^{* * *}$ $\mathrm{P}<0.001$ vs control group, ${ }^{\text {P }}<0.05$ vs PDT group.

Increased green and red fluorescence was found in the PDT group, and attenuated by NAC (Fig. S2A, B). Moreover, the co-localization of Lamp2 and LC3 induced by PDT was inhibited by NAC (Fig. S2C).

UCNPs-Ce6-mediated PDT inhibited the expression and secretion of pro-inflammatory factors through the regulation of ROS in M1 peritoneal macrophages

To investigate the relationship between ROS and pro-inflammatory cytokines, the protein expression levels of iNOS, IL-12, and TNF- $\alpha$ and the pro-inflammatory factors in the supernatants were examined by pretreatment with NAC. The results indicated that, compared with the PDT alone group, the expression and secretion of pro-inflammatory factors clearly increased with NAC pretreatment (Fig. 7A, B). We then examined the specific fluorescence markers CD16/32 and TNF- $\alpha$ of M1 peritoneal macrophages and found that the intensity of green fluorescence markers also increased significantly in the PDT-NAC group (Fig. 7C). These experiments indicated that PDT inhibited the expression and secretion of pro-inflammatory factors through the regulation of ROS in M1 peritoneal macrophages. 
Fig. 5. PDT induced autophagy through the PI3K/AKT/mTOR signaling pathway. (A) The expression levels of p-AKT, p-mTOR, and LC3 II/LC3 I at various times after PDT $(n=10)$. (B) The effect of LY294002 on the expressions levels of p-AKT, p-mTOR, and LC3 II/LC3 I after PDT ( $\mathrm{n}=10$ ). (C) The effect of triciribine on the expressions levels of p-AKT, p-mTOR, and LC3 II/LC3 I after PDT ( $\mathrm{n}=$ 10). ${ }^{*} \mathrm{P}<0.05$, ${ }^{* *} \mathrm{P}<0.01$ vs control group, ${ }^{~} \mathrm{P}<0.05$ vs PDT group.

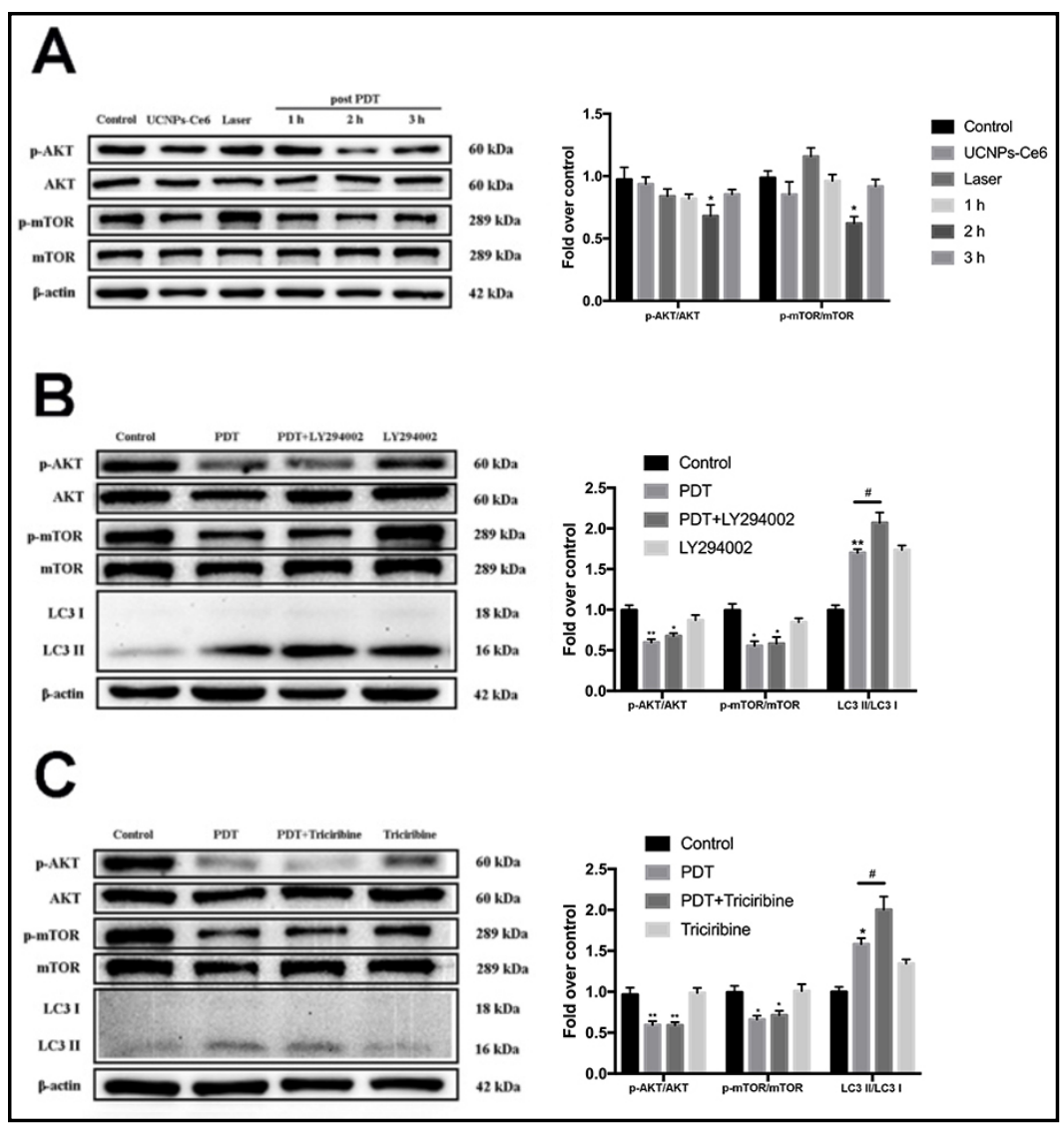

\section{Discussion}

In this study, ROS generated by UCNPs-Ce6-mediated PDT inhibited the expression of pro-inflammatory factors by inducing autophagy in M1 peritoneal macrophages. In addition, the PI3K/AKT/mTOR signaling pathway was involved in PDT-activated autophagy. Thus, our findings revealed an anti-inflammatory effect through autophagy induced by PDT, suggesting that UCNPs-Ce6-mediated PDT is a potential treatment for atherosclerosis.

ROS are crucial mediators of inflammation. It has been found that ROS can induce cell apoptosis and autophagy by regulating multiple signaling pathways, which plays an important role in various diseases [31-33]. Similarly, ROS, as products of PDT, have important biological effects in the treatment of cardiovascular disease $[2,5]$. In the present study, a significant increase in ROS generation was detected by flow cytometry and DCFH-DA in the PDT group, demonstrating that PDT was capable of producing ROS, which was clearly inhibited by NAC. Furthermore, our results indicated that the generation of PDT-induced ROS could activate autophagy in M1 peritoneal macrophages, which was also restrained by NAC. It was reported that ATG4 was identified as an effector molecule of ROS to promote LC3 lipogenesis, which is a key step of autophagy initiation [34]. Additional studies should be performed to verify it. In addition, previous reports have shown that ROS can not only inhibit but also promote the expression of pro-inflammatory cytokines through multiple signaling pathways [35-37]. In our experiments, ROS induced by PDT clearly inhibited the expression of pro-inflammatory factors. Interestingly, our previous studies verified that ROS generated by $1.0 \mathrm{~W} / \mathrm{cm}^{2}$ and 8 $\mu \mathrm{g} / \mathrm{mL}$ UCNPs-Ce6-PDT induced autophagy in THP-1 macrophage-derived foam cells and $2.0 \mathrm{~W} / \mathrm{cm}^{2}$ and $16 \mu \mathrm{g} / \mathrm{mL}$ UCNPs-Ce6-PDT induced apoptosis in THP-1 macrophages [5, 24]. 
Fig. 6. ROS generated by PDT induced autophagy via the P I 3 K / A K T / m T O R signaling pathway. (A) Intracellular ROS generation of M1 peritoneal $\mathrm{m}$ a c r o p h a g e $\mathrm{s}$ measured by DCFH-DA staining (scale bar: 50 $\mu \mathrm{m})(\mathrm{n}=10)$. (B) ROS generation measured by flow cytometry ( $\mathrm{n}=7$ ). (C) Western blotting analysis of the expression levels of p62, Beclin1, and LC3 II/LC3 I at $2 \mathrm{~h}$ after PDT $(\mathrm{n}=3)$. (D) Expression levels of p-AKT, and p-mTOR after PDT were analyzed by western blotting ( $\mathrm{n}=$ 3). ${ }^{*} \mathrm{P}<0.05$, ** $\mathrm{P}<0.01$, *** $\mathrm{P}<0.001$ vs control group, \# $\mathrm{P}<0.05$, \#\# $\mathrm{P}<0.01$, \#\#\# $\mathrm{P}<0.001$ vs PDT group.

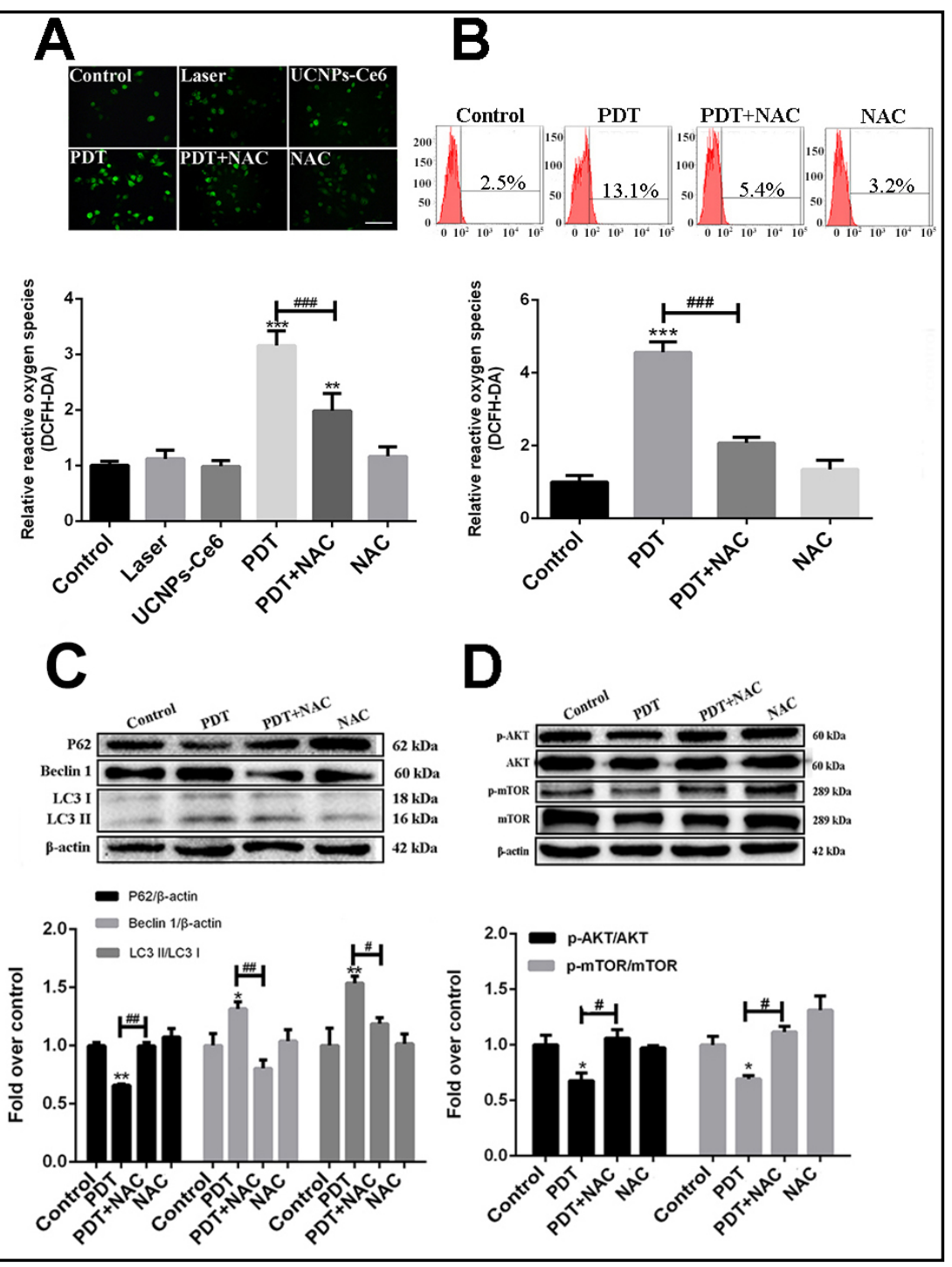

In the present study, we verified that ROS generated by $1.5 \mathrm{~W} / \mathrm{cm}^{2}$ and $6 \mu \mathrm{g} / \mathrm{mL}$ UCNPsCe6-PDT induced autophagy in M1 peritoneal macrophages. Therefore, distinct degrees of ROS produced by different intensities of laser may play diverse roles in the treatment of atherosclerosis.

Autophagy is a complex physiological process, and is indispensable for the reconstruction, regeneration, and repair of cells, as well as for the maintenance of cellular and organismal homeostasis [38-40]. Autophagy also play an important role in cardiovascular system, with autophagy dysfunction-contributing to the development of cardiovascular diseases such as atherosclerosis [41-43]. In this study, we found that PDT induced autophagy in a time-dependent manner, autophagy peaked at $2 \mathrm{~h}$, and these results were blocked by 3-MA. Moreover, typical autophagosomes with a phospholipid bilayer was found in the PDT group. MDC and acridine orange staining as well as the co-localization of LC3 and Lamp2 confirmed that autophagy was induced by PDT. Next, previous studies have confirmed that autophagy can promote cholesterol efflux, reduce lipid accumulation and inhibit the secretion of inflammatory factors in THP-1 macrophages [5, 36, 44]. In our study, PDT also inhibited the expression and secretion of pro-inflammatory factors in M1 peritoneal macrophage, and these effects were inhibited by 3-MA. Our results show that PDT inhibited the expression of proinflammatory factors by inducing autophagy in M1 peritoneal macrophage, which is consistent 


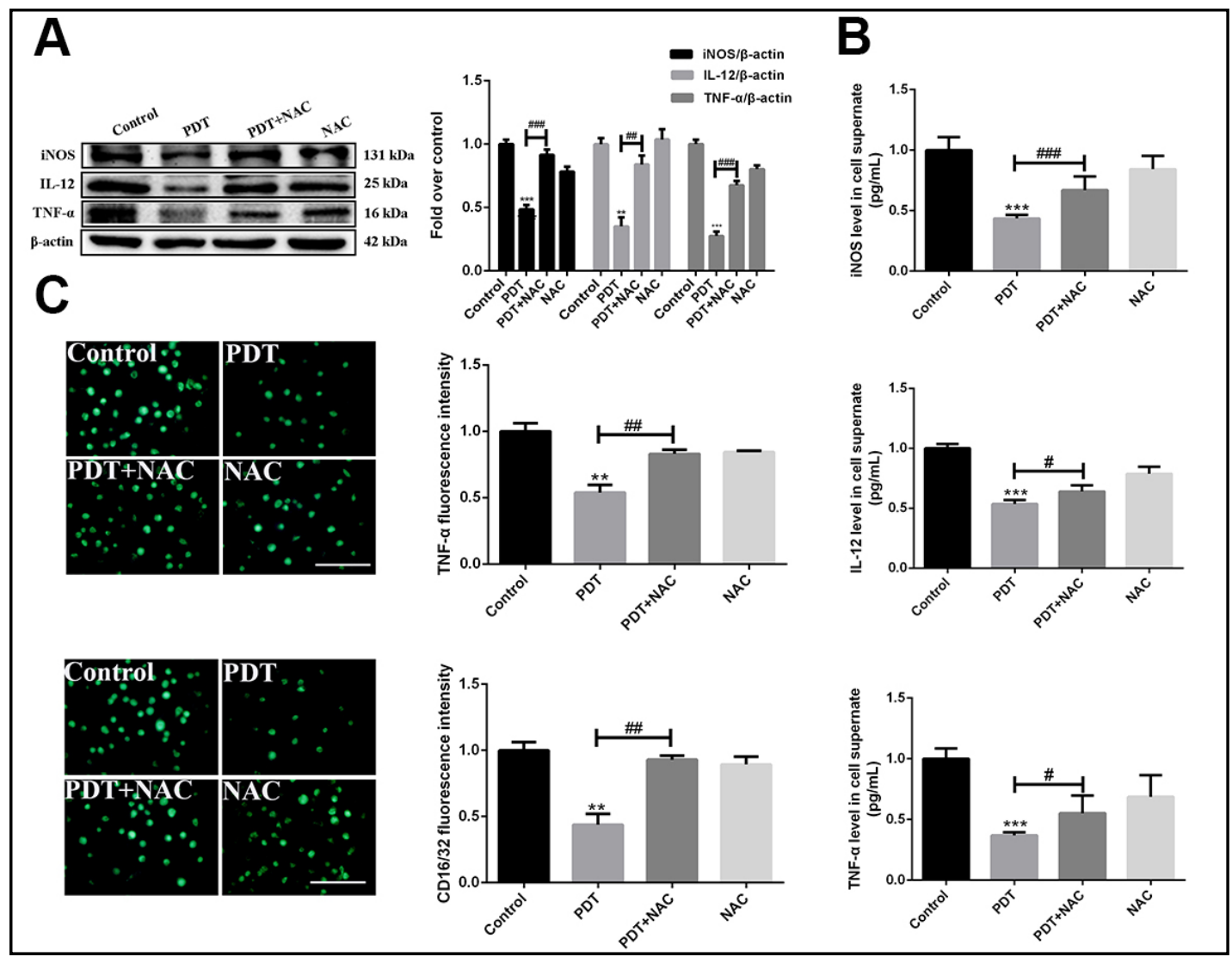

Fig. 7. ROS generated by PDT inhibited the expression of pro-inflammatory factors. (A) Western blotting analysis of the expression levels of iNOS, IL-12, and TNF- $\alpha$ at $12 \mathrm{~h}$ after PDT ( $\mathrm{n}=10)$. (B) Secretion levels of iNOS, IL-12, and TNF- $\alpha$ in cell supernatants were analyzed by western blotting $(\mathrm{n}=10)$. (C) Effect of NAC on the pro-inflammatory factors TNF- $\alpha$ and CD16/32 $12 \mathrm{~h}$ after different treatments by fluorescence assay (scale bar: $50 \mu \mathrm{m})(\mathrm{n}=10) .{ }^{*} \mathrm{P}<0.05$, ${ }^{* *} \mathrm{P}<0.01$, ${ }^{* * *} \mathrm{P}<0.001$ vs control group, ${ }^{\#} \mathrm{P}<0.05$, \#\# $\mathrm{P}<0.01$, \#\#\# $\mathrm{P}<0.001$ vs PDT group.

with studies of autophagy for the suppression of inflammation [45, 46]. It was reported that Toll-like receptor 4 (TLR4) was believed as a potential target to govern the pro-inflammatory reaction through autophagy [47]. Future study should be performed to better understand how autophagy induced by PDT inhibit the expression of pro-inflammatory factors.

Inhibition of the PI3K/AKT/ mTOR signaling pathway could induce autophagy and enhance the expression of autophagy-

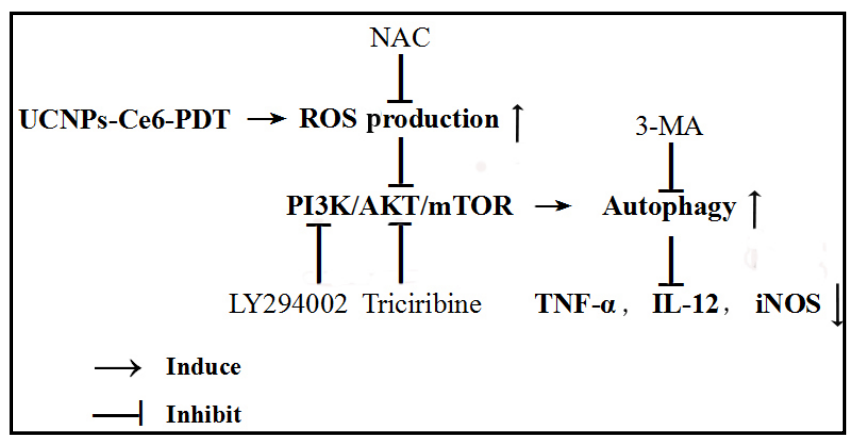

Fig. 8. Schematic diagram of the proposed mechanism. UCNPs-Ce6-mediated PDT activated autophagy by inhibiting the PI3K/AKT/mTOR signaling pathway regulated by ROS, which inhibited inflammation polarization of M1 peritoneal macrophages. related proteins [48-50]. In the present study, p-AKT, p-mTOR, and LC3II/LC3I were detected by pretreatment with the PI3K inhibitor LY294002 and AKT inhibitor triciribine, p-AKT and p-mTOR were not affected by LY294002 and triciribine, while LC3 II/LC3 I conversion clearly increased. Furthermore, 


\section{Cellular Physiology Cell Physiol Biochem 2019;52:1325-1338

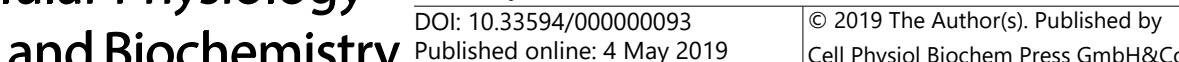 \\ \begin{tabular}{l|l} 
Published online: 4 May 2019 & Cell Physiol Biochem Press GmbH\&Co. KG
\end{tabular} \\ Han et al.: Upconversion Nanoparticle-Mediated Photodynamic Therapy Induces \\ Autophagy in M1 Peritoneal Macrophage}

the expression of p-AKT and p-mTOR increased with NAC pretreatment. These results demonstrated that autophagy was induced by PDT through the PI3K/AKT/mTOR signaling pathway via ROS generation in M1 peritoneal macrophages.

As illustrated in Fig. 8, these experiments confirmed that the UCNPs-Ce6-PDT enhanced the generation of ROS in M1 peritoneal macrophages, and the ROS induced autophagy through suppressing PI3K/AKT/mTOR signaling pathway. Furthermore, autophagy induced by ROS inhibited the expression of pro-inflammatory factors in M1 peritoneal macrophages.

\section{Conclusion}

ROS generated by UCNPs-Ce6-mediated PDT inhibited the expression of proinflammatory factors by activating autophagy via the PI3K/AKT/mTOR signaling pathway in M1 peritoneal macrophages.

\section{Acknowledgements}

This study was supported by the Foundation of Key Laboratories of Myocardial Ischemia Mechanism and Treatment (Harbin Medical University) (KF201814) and the Wu Liande Youth Science Foundation of Harbin Medical University (WLD-QN1104).

\section{Disclosure Statement}

The authors declare that there are no conflicts of interest.

\section{References}

1 Hansson GK, Hermansson A: The immune system in atherosclerosis. Nat Immunol 2011;12:204-212.

-2 Apostolakis S, Spandidos D: Chemokines and atherosclerosis: focus on the CX3CL1/CX3CR1 pathway. Acta Pharmacol Sin 2013;34:1251-1256.

3 Moore KJ, Sheedy FJ, Fisher EA: Macrophages in atherosclerosis: a dynamic balance. Nat Rev Immunol 2013;13:709-721.

4 Randolph GJ: Mechanisms that regulate macrophage burden in atherosclerosis. Circ Res 2014;114:17571771.

-5 Matthijsen RA, de Winther MP, Kuipers D, van der Made I, Weber C, Herias MV, Gijbels MJ, Buurman WA: Macrophage-specific expression of mannose-binding lectin controls atherosclerosis in low-density lipoprotein receptor-deficient mice. Circulation 2009;119:2188-2195.

-6 Tabas I, Bornfeldt KE: Macrophage Phenotype and Function in Different Stages of Atherosclerosis. Circ Res 2016;118:653-667.

7 Levine B, Kroemer G: Autophagy in the pathogenesis of disease. Cell 2008;132:27-42.

-8 Levine B, Mizushima N, Virgin HW: Autophagy in immunity and inflammation. Nature 2011;469:323-335.

-9 Netea-Maier RT, Plantinga TS, van de Veerdonk FL, Smit JW, Netea MG: Modulation of inflammation by autophagy: Consequences for human disease. Autophagy 2016;12:245-260.

-10 Nakatogawa H, Suzuki K, Kamada Y, Ohsumi Y: Dynamics and diversity in autophagy mechanisms: lessons from yeast. Nat Rev Mol Cell Biol 2009;10:458-467.

11 Nussenzweig SC, Verma S, Finkel T: The role of autophagy in vascular biology. Circ Res 2015;116:480-488.

12 Gatica D, Chiong M, Lavandero S, Klionsky DJ: Molecular mechanisms of autophagy in the cardiovascular system. Circ Res 2015;116:456-467.

13 Martinet W, De Meyer GR: Autophagy in atherosclerosis: a cell survival and death phenomenon with therapeutic potential. Circ Res 2009;104:304-317. 


\section{Cellular Physiology Cell Physiol Biochem 2019;52:1325-1338 \begin{tabular}{l|l} 
and Bincl $10.33594 / 000000093$ & 2019 The Author(s). Published by
\end{tabular} and BIOChemistry Published online: 4 May $2019 \quad$ Cell Physiol Biochem Press GmbH\&Co. KG \\ Han et al.: Upconversion Nanoparticle-Mediated Photodynamic Therapy Induces \\ Autophagy in M1 Peritoneal Macrophage}

14 Razani B, Feng C, Coleman T, Emanuel R, Wen H, Hwang S, Ting JP, Virgin HW, Kastan MB, Semenkovich CF: Autophagy links inflammasomes to atherosclerotic progression. Cell Metab 2012;15:534-544.

15 Schrijvers DM, De Meyer GR, Martinet W: Autophagy in atherosclerosis: a potential drug target for plaque stabilization. Arterioscler Thromb Vasc Biol 2011;31:2787-2791.

16 Castano AP, Mroz P, Hamblin MR: Photodynamic therapy and anti-tumour immunity. Nat Rev Cancer 2006;6:535-545.

17 Tan J, Sun C, Xu K, Wang C, Guo J: Immobilization of ALA-Zn(II) Coordination Polymer Pro-photosensitizers on Magnetite Colloidal Supraparticles for Target Photodynamic Therapy of Bladder Cancer. Small 2015;11:6338-6346.

18 Andrzejak M, Price M, Kessel DH: Apoptotic and autophagic responses to photodynamic therapy in 1c1c7 murine hepatoma cells. Autophagy 2011;7:979-984.

-19 Garg AD, Dudek AM, Ferreira GB, Verfaillie T, Vandenabeele P, Krysko DV, Mathieu C, Agostinis P: ROSinduced autophagy in cancer cells assists in evasion from determinants of immunogenic cell death. Autophagy 2013;9:1292-1307.

20 Abrahamse H, Hamblin MR: New photosensitizers for photodynamic therapy. Biochem J 2016;473:347364.

- 21 Kou J, Dou D, Yang L: Porphyrin photosensitizers in photodynamic therapy and its applications. Oncotarget 2017;8:81591-81603.

22 Wang C, Cheng L, Liu Z: Drug delivery with upconversion nanoparticles for multi-functional targeted cancer cell imaging and therapy. Biomaterials 2011;32:1110-1120.

-23 Yang K, Xu H, Cheng L, Sun C, Wang J, Liu Z: In vitro and in vivo near-infrared photothermal therapy of cancer using polypyrrole organic nanoparticles. Adv Mater 2012;24:5586-5592.

- 24 Zhu X, Wang H, Zheng L, Zhong Z, Li X, Zhao J, Kou J, Jiang Y, Zheng X, Liu Z, Li H, Cao W, Tian Y, Wang Y, Yang L: Upconversion nanoparticle-mediated photodynamic therapy induces THP-1 macrophage apoptosis via ROS bursts and activation of the mitochondrial caspase pathway. Int J Nanomedicine 2015;10:3719-3736.

25 Peng C, Li Y, Liang H, Cheng J, Li Q, Sun X, Li Z, Wang F, Guo Y, Tian Z, Yang L, Tian Y, Zhang Z, Cao W: Detection and photodynamic therapy of inflamed atherosclerotic plaques in the carotid artery of rabbits. J Photochem Photobiol B 2011;102:26-31.

26 Han XB, Li HX, Jiang YQ, Wang H, Li XS, Kou JY, Zheng YH, Liu ZN, Li H, Li J, Dou D, Wang Y, Tian Y, Yang LM: Upconversion nanoparticle-mediated photodynamic therapy induces autophagy and cholesterol efflux of macrophage-derived foam cells via ROS generation. Cell Death Dis 2017;8:e2864.

- 27 Wang H, Han RL, Yang LM, Shi JH, Liu ZJ, Hu Y, Wang Y, Liu SJ, Gan Y: Design and Synthesis of Core-ShellShell Upconversion Nanoparticles for NIR-Induced Drug Release, Photodynamic Therapy, and Cell Imaging. ACS Appl Mater Interfaces 2016;8:4416-4423.

28 Qiao XF, Zhou JC, Xiao JW, Wang YF, Sun LD, Yan CH: Triple-functional core-shell structured upconversion luminescent nanoparticles covalently grafted with photosensitizer for luminescent, magnetic resonance imaging and photodynamic therapy in vitro. Nanoscale 2012;4:4611-4623.

- 29 Kumar S, Guru SK, Pathania AS, Manda S, Kumar A, Bharate SB, Vishwakarma RA, Malik F, Bhushan S: Fascaplysin induces caspase mediated crosstalk between apoptosis and autophagy through the inhibition of PI3K/AKT/mTOR signaling cascade in human leukemia HL-60 cells. J Cell Biochem 2015;116:985-997.

- 30 Ricchelli F, Sileikyte J, Bernardi P: Shedding light on the mitochondrial permeability transition. Biochim Biophys Acta 2011;1807:482-490.

-31 Hui KF, Yeung PL, Chiang AK: Induction of MAPK- and ROS-dependent autophagy and apoptosis in gastric carcinoma by combination of romidepsin and bortezomib. Oncotarget 2016;7:4454-4467.

32 Gallagher LE, Williamson LE, Chan EY: Advances in Autophagy Regulatory Mechanisms. Cells 2016;5:pii:E24.

33 Zheng X, Wu J, Shao Q Li X, Kou J, Zhu X, Zhong Z, Jiang Y, Liu Z, Li H, Tian Y, Yang L: Apoptosis of THP-1 Macrophages Induced by Pseudohypericin-Mediated Sonodynamic Therapy Through the MitochondriaCaspase Pathway. Cell Physiol Biochem 2016;38:545-557.

- 34 Zhang X, Cheng X, Yu L: MCOLN1 is a ROS sensor in lysosomes that regulates autophagy. Nature Communications 2016;7:12109.

- 35 Lee IT, Yang CM: Role of NADPH oxidase/ROS in pro-inflammatory mediators-induced airway and pulmonary diseases. Biochem Pharmacol 2012;84:581-590. 


\section{Cellular Physiology Cell Physiol Biochem 2019;52:1325-1338 \begin{tabular}{cc|c|c|}
\hline DOl: 10.33594/000000093 & ( 2019 The Author(s). Published by \\
\hline
\end{tabular} and Biochemistry Published online: 4 May 2019 Cell Physiol Biochem Press GmbH\&Co. KG \\ Han et al.: Upconversion Nanoparticle-Mediated Photodynamic Therapy Induces \\ Autophagy in M1 Peritoneal Macrophage}

-36 Costley D, Mc Ewan C, Fowley C, McHale AP, Atchison J, Nomikou N, Callan JF: Treating cancer with sonodynamic therapy: a review. Int J Hyperthermia 2015;31:107-117.

37 Jiang Y, Kou J, Han X, Li X, Zhong Z, Liu Z, Zheng Y, Tian Y, Yang L: ROS-Dependent Activation of Autophagy through the PI3K/Akt/mTOR Pathway Is Induced by Hydroxysafflor Yellow A-Sonodynamic Therapy in THP-1 Macrophages. Oxid Med Cell Longev 2017;2017:8519169.

38 Boya P, Reggiori F, Codogno P: Emerging regulation and functions of autophagy. Nat Cell Biol 2013;15:713720.

39 Fan B, Zhang X, Ma Y, Zhang A: Fangchinoline Induces Apoptosis, Autophagy and Energetic Impairment in Bladder Cancer. Cell Physiol Biochem 2017;43:1003-1011.

40 Cao Z, Zhang H, Cai X, Fang W, Chai D, Wen Y, Chen H, Chu F, Zhang Y: Luteolin Promotes Cell Apoptosis by Inducing Autophagy in Hepatocellular Carcinoma. Cell Physiol Biochem 2017;43:1803-1812.

41 Ren J, Taegtmeyer H: Too much or not enough of a good thing--The Janus faces of autophagy in cardiac fuel and protein homeostasis. J Mol Cell Cardiol 2015;84:223-226.

42 Zhang Y, Sowers JR, Ren J: Targeting autophagy in obesity: from pathophysiology to management. Nat Rev Endocrinol 2018;14:356-376.

43 Wang S, Chen X, Nair S, Sun D, Wang X, Ren J: Deletion of protein tyrosine phosphatase 1B obliterates endoplasmic reticulum stress-induced myocardial dysfunction through regulation of autophagy. Biochim Biophys Acta Mol Basis Dis 2017;12:3060-3072.

- 44 Kou JY, Li Y, Zhong ZY, Jiang YQ, Li XS, Han XB, Liu ZN, Tian Y, Yang LM: Berberine-sonodynamic therapy induces autophagy and lipid unloading in macrophage. Cell Death Dis 2017;8:e2558.

- 45 Zhong Z, Sanchez-Lopez E, Karin M: Autophagy, Inflammation, and Immunity: A Troika Governing Cancer and Its Treatment. Cell 2016;166:288-298.

- 46 Fan X, Wang J, Hou J, Lin C, Bensoussan A, Chang D, Liu J, Wang B: Berberine alleviates ox-LDL induced inflammatory factors by up-regulation of autophagy via AMPK/mTOR signaling pathway. J Transl Med 2015;13:92.

47 Wang S, Zhu X, Xiong L, Zhang Y, Ren J: Toll-Like receptor 4 knockout alleviates paraquat-induced cardiomyocyte contractile dysfunction through an autophagy-dependent. mechanism. Toxicol Lett 2016;257:11-22.

48 Deretic V, Saitoh T, Akira S: Autophagy in infection, inflammation and immunity. Nat Rev Immunol 2013;13:722-737.

- 49 Janku F, Wheler JJ, Westin SN, Moulder SL, Naing A, Tsimberidou AM, Fu S, Falchook GS, Hong DS, GarridoLaguna I, Luthra R, Lee JJ, Lu KH, Kurzrock R: PI3K/AKT/mTOR inhibitors in patients with breast and gynecologic malignancies harboring PIK3CA mutations. J Clin Oncol 2012;30:777-782.

-50 Samarin J, Laketa V, Malz M, Roessler S, Stein I, Horwitz E, Singer S, Dimou E, Cigliano A, Bissinger M, Falk CS, Chen X, Dooley S, Pikarsky E, Calvisi DF, Schultz C, Schirmacher P, Breuhahn K: PI3K/AKT/mTORdependent stabilization of oncogenic far-upstream element binding proteins in hepatocellular carcinoma cells. Hepatology 2016;63:813-826. 